\title{
Towards a logic for reasoning about learning in a changing world
}

\author{
Prakash Panangaden* \\ School of Computer Science. McGill Universitv. Montréal,Canada \\ prakashecs.mcgill.cá \\ and Mehrnoosh Sadrzadeh ${ }^{\dagger}$ \\ Oxford University Computing Laboratory, Oxford, UK \\ mehrsecomlab.ox.ac.uk
}

\begin{abstract}
We develop an algebraic modal logic that combines epistemic and dynamic modalities with a view to modelling information acquisition (learning) by automated agents in a changing world. Unlike most treatments of dynamic epistemic logic, we have transitions that "change the state" of the underlying system and not just the state of knowledge of the agents. The key novel feature that emerges is the need to have a way of "inverting transitions" and distinguishing between transitions that "really happen" and transitions that are possible.

Our approach is algebraic, rather than being based on a Kripke-style semantics. The semantics are given in terms of quantales. We introduce a class of quantales with the appropriate inverse operations and use it to model toy robot-navigation problems, which illustrate how an agent learns information by taking actions. We discuss how a sound and complete logic of the algebra may be obtained from the positive fragment of PDL with converse.
\end{abstract}

\section{Introduction}

Epistemic logic has proved very important in the analysis of protocols in distributed systems (see, for example, [FHMV95]) and, more generally in any situation where there is some notion of cooperation or "agreement" between agents. The original work in distributed systems, by Halpern and Moses [HM84, HM90] and several others modelled the knowledge of agents using Kripke-style [Kri63] models. In these models there are a set of states (often called "possible worlds") in which the agent could be and, for each agent, an equivalence relation on the states. If two states are equivalent to an agent then that agent cannot "tell them apart". An agent "knows" a fact $\phi$ in the state $s$ if, in all states $t$ that the agent "thinks" are equivalent to $s$, the fact $\phi$ holds. The quoted words in the preceding sentences are, of course, unnecessary anthropomorphisms that are intended to give an intuition for the definitions.

A vital part of any analysis is how processes "learn" as they participate in protocols. The bulk of papers in the distributed systems community treat this as a change in the Kripke equivalence relations and argue about these changes only in the semantics. The logic itself does not have the "dynamic" modalities that refer to updating of the state of knowledge. On the other hand, dynamic epistemic logic (DEL) has indeed been studied; see, for example the recent book [vDvdHK08]. In the second author's doctoral dissertation an algebraic approach to dynamic epistemic logic was studied in depth [Sad06].

The advantage of working in the algebraic setting is that it abstracts over the details of the Kripke structures and showcases the high level structure of the actions and their updates. As a result, one can relate the structure of epistemic actions and their updates to the other areas of computer science, e.g. reasoning about correctness of programs and observational logics, e.g. of Abramsky and Vickers [AV93].

\footnotetext{
*Supported by NSERC and the Office of Naval Research.

${ }^{\dagger}$ Corresponding author; supported by EPSRC grant EP/F042728/1. A more algebraic version of this paper that does not include the logic part, is submitted to AMAST 2010.
} 
In particular, it turns out that the epistemic update is the action of the quantale of programs/actions on the module of propositions (factual and epistemic), hence it is the left adjoint to the dynamic modality which encodes the weakest precondition of Hoare Logic. Secondly (and in a novel attempt), epistemic modalities (too) are encoded as an adjoint pair: the belief modality is the right adjoint of the appearance map, which is the lifting (to subsets) of the accessibility relation of the Kripke structure. Apart from the conceptual novelty and the charm of this adjoint-based approach, it offers a very simple method of reasoning about knowledge acquisition after an action, i.e. by uniform unfolding of epistemic and dynamic adjunctions. This method simplifies, to a great extent, the proofs of complex protocols and puzzles, such as the muddy children, even the versions with dishonest children.

The bulk of the work in this area (algebraic and relational), concerns situations where the state of knowledge is changed by broadcasts but not situations where the state of the system is changed. An illuminating and concrete example of such situations arise in, but are not limited to, robot navigation in AI. The general features of these scenarios is that an agent is given the description of a place, but cannot determine where it is; however, it can move and as a result may acquire information that allows it to infer where it is. Consider a robot is given the map of a small computing laboratory with 5 rooms accessible via 3 actions, as follows:

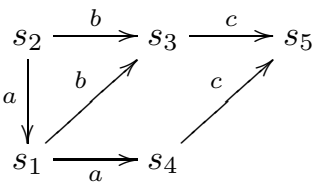

Since the robot can do the same actions in the pairs $s_{1}, s_{2}$ and $s_{3}, s_{4}$, it cannot tell them apart. Once in $s_{1}$ (similarly for $s_{2}$ ), it thinks that it could be in $s_{1}$ or $s_{2}$, and once in $s_{3}$ (similarly for $s_{4}$ ), it thinks that it could be in $s_{3}$ or $s_{4}$. But if once in $s_{1}$ it performs an $a$ action, then it reaches $s_{4}$ and learns where it is and where it was before moving.

A deeper investigation of this example reveals that it is not a question of "patching up" the theory. There are some interesting fundamental changes that need to be made. First of all, one has to distinguish between transitions that exist in the agent's "mental model" of the system and actions that actually occur. Second, one has to introduce a converse dynamic modality in order to correctly formulate the axioms for updating knowledge. To see why, let us reason as we think the robot should: when it reaches $s_{4}$, it checks with its map and reasons that the only way it could have reached $s_{4}$ would be that it was originally in $s_{1}$. It rules out $s_{3}$ from its uncertainty set about $s_{4}$, because, according to the map, it could not have reached $s_{3}$ via an $a$ action. We have two types of data here, the locations and actions described on the map versus the ones in reality. The data on the map are hard-coded in the robot and there is no uncertainty about it, the map fully describes the system. But the real locations and actions are only partially known. The robot is uncertain about locations and the actions it takes change its uncertainties. The other issue is that to be able to encode what actions could have led the robot to where it is, it needs to look back, so we need a converse operation to reason about the past. Now by moving from $s_{1}$ to $s_{4}$, the robot has changed its uncertainty, acquired information, and learned where it is located. This is exactly the manner in which our new uncertainty reduction axiom formalizes the elimination of past uncertainties: after performing a certain move in the real world, the robot consults its description, considers its possibilities and eliminates the ones that could not have been reached as a result of the action it just performed. Furthermore with this converse operation, we can also derive information about past, that the robot was in $s_{1}$ before doing action $a$.

This paper presents an algebraic theory with these features. The algebra of previous work, e.g. [BCS07] fails for these scenarios. The reason is that its reduction axiom responsible for changing the uncertainty 
after an action, is only geared towards epistemic actions and is not powerful enough for fact-changing actions. It requires that the uncertainty about (possible states of) a location after an action to be included in the result of applying the action to the uncertainty about the location beforehand, a property similar to perfect recall in protocol models of [HM84, HM90]. This fails here, since after performing an $a$ at $s_{1}$ one ends up in $s_{4}$, hence uncertainty about $s_{1}$ after an $a$ is the same as uncertainty about $s_{4}$, consisting of $s_{3}$ and $s_{4}$. But performing $a$ on the set of uncertainties about $s_{1}$, consisting of $s_{1}$ and $s_{2}$, results in both $s_{4}$ and $s_{1}$. However, $\left\{s_{4}, s_{1}\right\}$ is not included in $\left\{s_{3}, s_{4}\right\}$. Moreover, after the robot moved to $s_{4}$, it can conclude that it was in $s_{1}$ before moving; the language of [BCS07] simply cannot express these past tense properties.

Finally, regarding related work, Dynamic Epistemic Logic has been extended with assignments and post-conditions, e.g. see vanDit05], to be able to reason about learning after fact-changing actions. Although the scenarios we are interested in can be modeled in the relational models of [vanDit05] (these being transition systems with uncertainty as well as action transitions), the reduction axiom thereof cannot derive the knowledge properties we are interested in. This may be because their approach has different kinds of fact-changing actions in mind, e.g. the ones that change the status of a child in the muddy children puzzle from dirty to clean via washing (and not our location-changing actions). Nevertheless, they do not discuss or specify what kind of actions their reduction axiom targets. So there is indeed a gap in modeling and reasoning about the scenarios we deal with here. On a positive note, since we use converse actions, there might be connections to a DEL with converse actions, e.g. see [Auch07. However, an elementary study seems to indicate that our reduction axiom is still very different from the one developed there. A further exploration of these connections constitutes future work.

In what follows, we briefly review the algebraic setting which formalizes information acquisition from such navigation protocols and apply it to reason about learning from two toy examples. One of these examples is the map-based navigation scenario discussed above, and the other one is navigation on the grid, which is not based on a map. Further applications of our setting are to AI, mobile communication, security, and control theory. We show that our algebraic structure generalizes that of previous work [BCS07], by proving that the latter faithfully embeds in ours. Hence our setting is also strong enough to reason about learning as a result of communication actions. The slight contribution of this paper over its AMAST version is the presentation of a logic for part of the algebra. The full version of the paper is available from [PS10].

\section{The Algebra of di-Systems}

We need to model "actions" and "formulas". The actions are modelled by a quantale while the propositions are a module over the quantale; i.e. actions modify propositions.

Definition 2.1. A quantale $(Q, \bigvee, \bullet, 1)$ is a sup-lattice equipped with a unital monoid structure satisfying $q \bullet \bigvee_{i} q_{i}=\bigvee_{i}\left(q \bullet q_{i}\right)$ and $\bigvee_{i} q_{i} \bullet q=\bigvee_{i}\left(q_{i} \bullet q\right)$. Instead of an arbitrary sup-lattice we take it to be a completely distributive prime-algebraic lattice.

Recall that a prime element, or simply "prime", $p$ in lattice has the property that for any $x, y$ in the lattice, $p \leq x \vee y$ implies that $p \leq x$ or $p \leq y$; "prime algebraic" means that every element is the supremum of the primes below it. The restriction to prime algebraic lattices is not a serious restriction for the logical applications that we are considering; it would be a restriction for extensions to probabilistic systems; we will address such issues in future work. The use of algebraicity is to be able to use simple set-theoretic arguments via the representation theorem for such lattices [Win09]. For finite distributive lattices it is 
not a restriction at all because of Birkhoff's classical representation theorem. Henceforth, we will not explicitly state that we are working with (completely) distributive prime-algebraic lattices.

Definition 2.2. A right-module over $Q$ is a sup-lattice $M$ with an action of $Q$ on $M,-\cdot-: M \times Q$ $\rightarrow M$ satisfying

$$
(m \cdot q) \cdot q^{\prime}=m \cdot\left(q \bullet q^{\prime}\right) \quad m \cdot \bigvee_{i} q_{i}=\bigvee_{i}\left(m \cdot q_{i}\right) \quad \bigvee_{i} m_{i} \cdot q=\bigvee_{i}\left(m_{i} \cdot q\right) \quad m \cdot 1=m
$$

We call the collection of actions and propositions a system. We do not have an explicit notion of "state".

Definition 2.3. A system is a pair consisting of a quantale $Q$ and a right-module $M$ over $Q$. We write $(M, Q, \cdot)$ for a system.

This is closely related to a definition of Abramsky and Vickers who also studied quantales of actions, see AV93.

Since the action preserves all the joins of its module, the map $-\cdot q: M \rightarrow M$, obtained by fixing the module argument, has a Galois right adjoint that preserves all the meets. This is denoted by $-\cdot q \dashv[q]-$ and defined in the canonical way.

Proposition 2.4. The following inequalities hold in any system $(M, Q, \cdot)$ :

(1) $([q] m) \cdot q \leq m$

(3) $\quad\left(m \wedge m^{\prime}\right) \cdot q \leq m \cdot q \wedge m^{\prime} \cdot q$

(5) $\quad[q]\left(m \vee m^{\prime}\right) \geq[q] m \vee[q] m^{\prime}$

(6) $q \leq q^{\prime} \Longrightarrow\left[q^{\prime}\right] m \leq[q] m$

(8) $\left[q \vee q^{\prime}\right] m=[q] m \wedge\left[q^{\prime}\right] m$

(10) $\left[q \wedge q^{\prime}\right] m \geq[q] m \vee\left[q^{\prime}\right] m$

(12) $\left[\bigvee_{i} q_{i}\right] m=\bigwedge_{i}\left[q_{i}\right] m$
(2) $m \leq[q](m \cdot q)$

(4) $m \cdot\left(q \wedge q^{\prime}\right) \leq m \cdot q \wedge m \cdot q^{\prime}$

(7) $\quad[\perp] m=\top$

(9) $\left[q \vee q^{\prime}\right] m \leq[q] m \vee\left[q^{\prime}\right] m$

(11) $\left[q \wedge q^{\prime}\right] m \geq[q] m \wedge\left[q^{\prime}\right] m$

Proof. (1) and (2) are immediate consequnces of the definition of $[q]$ as a right adjoint. (3), (4), (5) follow from monotonicity. For (6), assume $q \leq q^{\prime}$ and we have to show

$$
\bigvee\left\{m^{\prime} \mid m^{\prime} \cdot q^{\prime} \leq m\right\} \leq \bigvee\left\{m^{\prime \prime} \mid m^{\prime \prime} \cdot q \leq m\right\}
$$

It suffices to show that an arbitrary element of the lhs set is in the rhs set. Take one such element $m^{\prime}$, we have $m^{\prime} \cdot q^{\prime} \leq m$, but since $q \leq q^{\prime}$, we also have that $m^{\prime} \cdot q \leq m^{\prime} \cdot q^{\prime}$, hence $m^{\prime} \cdot q \leq m$, i.e. $m^{\prime}$ is also in the rhs set. For (7), the direction $[\perp] m \leq \top$ is trivial, the other direction $T \leq[\perp] m$ is equivalent to $T \cdot \perp \leq m$, which holds since $T \cdot \perp=\perp$. For (8), the $\leq$ direction follows from (6) and definition of meet, for the $\geq$ direction we have to show $[q] m \wedge\left[q^{\prime}\right] m \leq\left[q \vee q^{\prime}\right] m$, which is by adjunction equivalent to $\left([q] m \wedge\left[q^{\prime}\right] m\right) \cdot\left(q \vee q^{\prime}\right) \leq m$. By join preservation of action, this is equivalent to $\left([q] m \wedge\left[q^{\prime}\right] m\right) \cdot q \vee\left([q] m \wedge\left[q^{\prime}\right] m\right) \cdot q^{\prime} \leq m$. To show this, we have to show that both disjuncts are less than or equal to $m$. Consider the first one, by (3) and transitivity, it suffices to show $[q] m \cdot q \wedge\left[q^{\prime}\right] m \cdot q \leq m$, by the definition of meet and transitivity it suffices to show either of the conjuncts satisfy the inequality, now $[q] m \cdot q \leq m$, is true by (1). The proofs of the remaining items follow from these in a similar way, e.g. (12) follows from (7) and (8).

Definition 2.5. A sup lattice $M$ is a right di-module of the quantale $Q$ whenever there are two right actions $-\cdot-: M \times Q \rightarrow M$ and $-\times-: M \times Q \rightarrow M$. We call the pair of a quantale and its di-module $(M, Q, \cdot, \times)$ a di-System. 
Definition 2.6. Whenever the two actions - written $\cdot$ and $\cdot^{-1}$ for the purposes of this definition - of a di-system are related by the following three axioms

$$
\begin{array}{lll}
\text { (i) } & m \cdot q \leq m^{\prime} \Longrightarrow m \leq m^{\prime} \cdot{ }^{-1} q & \text { whenever } m \cdot q \neq \perp \\
\text { (ii) } & m \cdot{ }^{-1} q \leq m^{\prime} \Longrightarrow m \leq m^{\prime} \cdot q & \text { whenever } m \cdot{ }^{-1} q \neq \perp \\
\text { (iii) } & m \cdot{ }^{-1}\left(q \bullet q^{\prime}\right)=\left(m \cdot{ }^{-1} q^{\prime}\right) \cdot{ }^{-1} q &
\end{array}
$$

then we refer to the di-system as a converse di-System and denote it by $\left(M, Q, \cdot, \cdot^{-1}\right)$.

Proposition 2.7. A converse di-System satisfies $m \leq(m \cdot q) \cdot^{-1} q$, whenever $m \cdot q \neq \perp$, and $m \leq$ $\left(m \cdot{ }^{-1} q\right) \cdot q$, whenever $m \cdot \cdot^{-1} q \neq \perp$.

Definition 2.8. A converse di-System is past-deterministic iff $m \leq m^{\prime} \cdot q \Longrightarrow m \cdot{ }^{-1} q \leq m^{\prime}$, for $m^{\prime} \cdot q \neq \perp$. It is future-deterministic iff $m \leq m^{\prime} \cdot{ }^{-1} q \Longrightarrow m \cdot q \leq m^{\prime}$, for $m^{\prime} \cdot{ }^{-1} q \neq \perp$.

Proposition 2.9. In a past-deterministic converse di-System we have $m \leq m^{\prime} \cdot q \Longleftrightarrow m^{\cdot-1} q \leq m^{\prime}$ for $m^{\prime} \cdot q, m \cdot{ }^{-1} q \neq \perp$ and in a future-deterministic converse di-System we have $m \leq m^{\prime} \cdot{ }^{-1} q \Longleftrightarrow$ $m \cdot q \leq m^{\prime}$ for $m^{\prime} \cdot{ }^{-1} q, m \cdot q \neq \perp$.

Example 2.10. Consider the following transition system

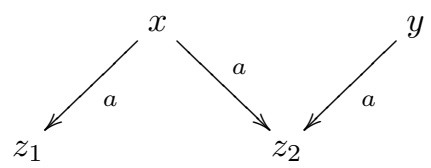

We model this as a di-System $\left(M, Q, \cdot \cdot^{-1}\right)$ by assuming $x, y, z_{1}, z_{2} \in M, a \in Q$, and $x \cdot a=z_{1} \vee$ $z_{2}, y \cdot a=z_{2}, z_{1} \cdot{ }^{-1} a=x$, and $z_{2} \cdot{ }^{-1} a=x \vee y$. It is easy to check that these satisfy the inequalities of definition 2.6. but not their converses: the transition system is neither past-deterministic nor futuredeterministic. A counterexample for the converse of part $(i)$ is $x \leq z_{2}{ }^{-1}$ a but $x \cdot a \notin z_{2}$. If we eliminate the leftmost edge, then the system becomes future-deterministic and the converse of $(i)$ holds. A counterexample for the converse of part $(i i)$ is $z_{2} \leq y \cdot a$ but $z_{2} \cdot{ }^{-1} a \leq y$. If we eliminate the rightmost edge, then the system becomes past-deterministic and the converse of $(i)$ holds.

Example 2.11. The powerset $\mathcal{P}(S)$ of a set $S$ is the right di-module of the quantale of all the relations thereon $\mathcal{P}(S \times S)$. Relational composition is the monoid multiplication, the diagonal relation is its unit, and the join is set union. The action and its converse are the point wise images of the relation and its converse, i.e. for $W \subseteq S$ and $R \subseteq S \times S$

$$
W \cdot R=\bigcup_{w \in W} R[w] \quad W \cdot^{-1} R=\bigcup_{w \in W} R^{-1}[w]
$$

It is easy to see that $W \cdot{ }^{-1} R=W \cdot R^{-1}$. If $R^{-1}$ is a singleton then this di-system becomes a pastdeterministic one, if $R$ is a singleton, it becomes future-deterministic.

The converse action preserves all the joins of the module, thus similar to the action, it has a Galois right adjoint denoted by $-\cdot^{-1} q \dashv[q]^{-1}-$, defined in the canonical way. It is worth observing that interaction axioms of, e.g. [Dun05] follow from our adjunction and converse axioms, in the following incremental order:

Proposition 2.12. In any converse di-System we have

$$
[q]\left(m \vee m^{\prime}\right) \leq[q] m \vee m^{\prime} \cdot{ }^{-1} q \quad[q]^{-1}\left(m \vee m^{\prime}\right) \leq[q]^{-1} m \vee m^{\prime} \cdot q
$$

Proposition 2.13. In a future-deterministic converse di-System we have

$$
m \cdot{ }^{-1} q \wedge[q] m^{\prime} \leq\left(m \wedge m^{\prime}\right) \cdot{ }^{-1} q
$$


Proposition 2.14. In a past-deterministic converse di-System we have

$$
m \cdot q \wedge[q]^{-1} m^{\prime} \leq\left(m \wedge m^{\prime}\right) \cdot q
$$

These four properties are meant to axiomatize de Morgan duality of $-\cdot q$ and $[q]^{-1}-$, also of $-\cdot^{-1} q$ and $[q]-$, in the absence of negation. In a Boolean module, these de Morgan dualities become explicit.

Proposition 2.15. If the module of a past and future deterministic converse di-System is a Boolean algebra with negation operator $\neg-: M \rightarrow M$, we have $m \cdot q=\neg[q]^{-1} \neg m$ and $m^{\cdot-1} q=\neg[q] \neg m$.

For details of this, we refer the reader to [PS10]. We have also defined a Kleene star for iteration and shown that it preserves the adjunctions. In the Boolean setting of [vK98], these iteration operators have been used to model modalities of temporal logic.

\section{Second Order di-Systems}

To distinguish the "potential" actions that happen in the mind of the agent, e.g. actions described by a map, from the "real actions", we go higher order. We make real actions act on the di-system that describes potential actions. Real actions change the state of the system as described by the map.

Definition 3.1. A second order di-System $\left(\mathcal{D}, Q^{\prime}, \odot, \otimes\right)$ is a di-System whose module is a di-System, $\mathcal{D}=(M, Q, \cdot, \times)$, i.e. $-\odot-: \mathcal{D} \times Q^{\prime} \rightarrow \mathcal{D}$ and $-\otimes-: \mathcal{D} \times Q^{\prime} \rightarrow \mathcal{D}$.

Real actions have an extra significance, they also change the uncertainties of (some of) the states. To encode the uncertainties, we use lax endomorphisms of the system. The reason these are called lax is that we require them to satisfy axiomatic inequalities (rather than equalities), namely inequalities (1) and (2) below . These axioms encode the change of uncertainty and the reason they are inequalities has been motivated in [Sad06]. In a nutshell, they are so to be able to encode the process of learning as a decrease in the uncertainty (hence an increase in information).

Definition 3.2. A lax endomorphism $u$ of a second order di-System consists of a pair of endomorphisms $u=\left(u^{M}: M \rightarrow M, u^{Q}: Q \rightarrow Q\right)$, where $u^{M}$ preserves joins of $M$ and $u^{Q}$ preserves joins of $Q$, moreover we have

$$
\begin{aligned}
(1) u^{M}(m \odot q) & \leq \bigvee\left\{m^{\prime} \in M \mid m^{\prime} \leq u^{M}(m \cdot q), \quad m^{\prime} \times u^{Q}(q) \neq \perp\right\} \\
(2) u^{Q}\left(q \bullet q^{\prime}\right) & \leq u^{Q}(q) \bullet u^{Q}\left(q^{\prime}\right), \quad \text { (3) } 1 \leq u^{Q}(1)
\end{aligned}
$$

Since each projection of $u$ is join preserving, it has a Galois right adjoint, we denote it by $\square=$ $\left(\square^{M}, \square^{Q}\right)$. These are canonically defined as follows

$$
\square^{M} m:=\bigvee\left\{m^{\prime} \in M \mid u^{M}\left(m^{\prime}\right) \leq m\right\} \quad \square^{Q} q:=\bigvee\left\{q^{\prime} \in Q \mid u^{Q}\left(q^{\prime}\right) \leq q\right\}
$$

Definition 3.3. A second order informative di-System $\left((M, Q,-\cdot-, \times), Q^{\prime}, \odot, \otimes, u\right)$ is a second order di-System endowed with a di-System lax endomorphism.

The second order Informative di-System that we need for applications are second order converse disystems. As we shall see in more details later, these will be a generalization of Epistemic Systems of [BCS07] to also include actions that are not necessarily epistemic. We refer to them as Navigation di-Systems, defined as follows 
Definition 3.4. A Navigation di-System (Nav-diSys) is a second order informative di-System, where $\left(M, Q, \cdot,{ }^{-1}\right)$ is a converse di-System, $u$ is a second order lax di-System endormorphism, and - $\odot-$ and $-\odot^{-1}$ are given by

$$
\begin{aligned}
-\odot-\quad: & \left(M, Q, \cdot, \cdot \cdot^{-1}\right) \times Q \rightarrow\left(M, Q, \cdot \cdot^{-1}\right) \\
-\odot^{-1}-\quad: & \left(M, Q, \cdot, \cdot \cdot^{-1}\right) \times Q \rightarrow\left(M, Q, \cdot \cdot^{-1}\right)
\end{aligned}
$$

The second order action $-\odot-$ does not change the base di-System, but changes the lax endomorpshisms via inequality (1) of definition 3.2. It can be read in an operational way as restricting $u^{M}(m \odot q)$ to elements in the uncertainty of its corresponding lower action, i.e. $u^{M}(m \cdot q)$, to the ones that can be reached via a $u^{Q}(q)$ action.

\subsection{Interpretation}

Given a Navigation di-System $\left.\left(M, Q, \cdot, \cdot^{-1}\right), Q, \odot, \odot^{-1}, u\right)$ we interpret elements of the module as propositions and the order as entailment, thus $m \vee m^{\prime}$ is the logical disjunction and $\perp$ is the falsum. The elements of the quantale are interpreted as actions and the order is the order of non-determinism, thus $q \vee q^{\prime}$ is the non-deterministic choice and $\perp$ is crash, monoid multiplication $q \bullet q^{\prime}$ is sequential composition, and its unit 1 is the action that does nothing. The actions of the base converse di-System are to be thought of as potential, e.g. descriptions of actions on a map, the actions of the second order di-System are their real counterparts. For simplicity, we make the two di-Systems share the same action labels that live in the quantale $Q$ and the higher order action to mimic their base counterparts.

As is usual, the real world comes with some uncertainties and the effect of performing the real actions is to remove some of these uncertainties. The uncertainties are modeled by the endomorphisms of the module and quantale. We read them as follows

- $u^{M}(m)$ is the uncertainty about proposition $m$, the join of all propositions that are possibly true when in reality $m$ is true. For example $u^{M}(m)=m \vee m^{\prime}$, says that in reality $m$ is true, but agent considers it possible that either $m$ or $m^{\prime}$ might be true.

- $u^{Q}(q)$ is the uncertainty about action $q$, the join of all actions that are possibly happening when in reality action $q$ is happening. E.g. $u^{Q}(q)=q \vee q^{\prime}$ says that in reality action $q$ is happening but the agent considers it possible that either $q$ or $q^{\prime}$ is.

We interpret the right adjoints to uncertainty in an operational manner as follows

- $\square^{M} m$ reads as 'according to the information available $m$ holds in reality'. Alternatively, one can use the belief modality of doxastic logic and read it as 'it is believed that $m$ holds in reality'.

- $\square^{Q} q$ reads 'according to the information available $q$ is happening in reality', or using the belief modality as 'it is believed that action $q$ is happening in reality'.

We refer to axiom (1) of definition 3.2 as the uncertainty reduction axiom. The intuition behind it is as follows: when one does actions in reality, they change our uncertainty. In navigation systems this change is as follows: the uncertainty after performing an action in reality $u^{M}(m \odot q)$ is the uncertainty of performing a potential action according to the description of the system, i.e. $u^{M}(m \cdot q)$ minus the choices to which one could not have reached via a $q$ action (according to the description). For example, $u^{M}(m \cdot q)$ can be a choice of $m^{\prime} \vee m^{\prime \prime}$ and it is not possible to reach $m^{\prime}$ via a $q$ action, i.e. $m^{\prime} \cdot{ }^{-1} q=\perp$. Hence $m^{\prime}$ is removed from the choices in $u^{M}(m \odot q)$, hence $u^{M}(m \odot q)=m^{\prime \prime}$. The other two 
inequalities are for coherence of uncertainty with regard to composition, the motivations for these are as in [BCS07].

\section{Applications to Navigation}

Navigation systems are described by a set of locations $S$, a set of atomic actions $A c$, and a set of possible movements $A c \subseteq S \times S$.

Definition 4.1. On a set of locations $S$ and a set of action labels $A c \subseteq S \times S$, a concrete Nav-diSys is

$$
\mathcal{N}=\left(\left(\mathcal{P}(S), \mathcal{P}(S \times S),-\cdot-,-\cdot^{-1}-\right), \mathcal{P}(S \times S),-\odot-,-\odot^{-1}-,\left(u^{M}, i d^{Q}\right)\right)
$$

where the actions $-\cdot-$ and $-\cdot^{-1}$ - are as defined as in example 2.11] and uncertainty maps are build from the image of indistinguishability of states under actions.

Finally, we abide to the convention of impossibility of lack of uncertainty, that if the agent can do an action at a location then it should be the case the he has some uncertainty at that location. This is because, agents move to be able to find out where they are, if they already know where they are, then there is no problem to be modeled.

\subsection{Map-based Navigation}

We encode the navigation protocol of introduction in a concrete Nav-diSys with set of locations $S=$ $\left\{s_{1}, s_{2}, s_{3}, s_{4}, s_{5}\right\}$, set of actions $A c=\{a, b, c\}$, and applicability of actions and uncertainty of states as described there, similar to example 2.10 We quotient this over the impossibility of lack of uncertainty and show that after doing an $a$ action on $s_{1}$, the robot knows where it is and was before.

Proposition 4.2. The following hold in a concrete $\mathcal{N} / \Theta$ based on the above data.

$$
s_{1} \leq[a] \square^{M} s_{4} \quad s_{1} \leq[a] \square^{M}[a]^{-1} s_{1}
$$

Proof. Consider the first one: by the adjunction $-\odot a \dashv[a]-$, it is equivalent to $s_{1} \odot a \leq \square^{M} s_{4}$. By the adjunction $u^{M} \dashv \square^{M}$, this is equivalent to $u^{M}\left(s_{1} \odot a\right) \leq s_{4}$. Now by the uncertainty reduction inequality, it is enough to show that

$$
\bigvee\left\{s_{i} \in S \mid s_{i} \leq u^{M}\left(s_{1} \cdot a\right), s_{i} \cdot{ }^{-1} a \neq \perp\right\} \leq s_{4}
$$

Since $s_{1} \cdot a=s_{4}$, and $u^{M}\left(s_{4}\right)=s_{3} \vee s_{4}$, but $s_{3} \cdot{ }^{-1} a=\perp$ where as $s_{4} \cdot{ }^{-1} a \neq \perp$, hence the lhs of the above is equal to $s_{4}$, which is $\leq s_{4}$. Consider the second inequality, it becomes equivalent to $u^{M}\left(s_{1} \odot a\right) \odot^{-1} a \leq s_{1}$, by a series of 3 unfoldings of adjunctions. We have shown that $u^{M}\left(s_{1} \odot a\right) \leq s_{4}$, so it suffices to show $s_{4} \odot^{-1} a \leq s_{1}$, which is true since $s_{4} \odot^{-1} a=s_{4} \cdot{ }^{-1} a=s_{1} \leq s_{1}$.

\subsection{Grid Navigation}

A typical robot navigation protocol is as follows, a robot is in a grid with $n$ rows and $m$ columns, it can go up, down, left, and right and is supposed to move about and find out where it is. The grid cells look alike to it as long as it can do the same movements in them, hence it knows where it is iff it ends up in one of the four corner cells. We model this protocol in a concrete Nav-diSys and show that no matter 
where the robot is, there is always some sequences of movements that it can do to get it to one of the corners. After doing either of these it learns where it is and where it was beforehand.

Each grid cell is modeled by a state $s_{i j}$ in the $i$ 'th row and $j$ 'th column. Uncertainty of corner states $s_{11}, s_{1 m}, s_{n 1}, s_{n m}$ is identity, i.e.

$$
u^{M}\left(s_{11}\right)=s_{11} \quad u^{M}\left(s_{1 m}\right)=s_{1 m} \quad u^{M}\left(s_{n 1}\right)=s_{n 1} \quad u^{M}\left(s_{n m}\right)=s_{n m}
$$

For the rest of the cells we have

$$
u^{M}\left(s_{i j}\right)=\bigvee_{1<x<n} s_{x y} \quad u^{M}\left(s_{1 j}\right)=\bigvee_{1<y<m} s_{1 y} \quad u^{M}\left(s_{i 1}\right)=\bigvee_{1<x<n} s_{x 1}
$$

The set of actions is $A c=\{u, d, l, r\}$, their non-applicability is as follows

$$
s_{1 j} \cdot u=s_{1 j} \cdot{ }^{-1} d=s_{i 1} \cdot l=s_{i 1} \cdot{ }^{-1} r=s_{n j} \cdot d=s_{n j} \cdot{ }^{-1} u=s_{i m} \cdot r=s_{i m} \cdot{ }^{-1} l=\perp
$$

All the other actions are applicable in all the other states.

Proposition 4.3. The following hold in a concrete $\mathcal{N}$ based on the above data.

$$
s_{i j} \leq[\alpha] \square\left(s_{11} \vee s_{1 m} \vee s_{n 1} \vee s_{n m}\right) \quad s_{i j} \leq[\alpha] \square[\alpha]^{-1} s_{i j}
$$

for $1<i<n, 1<j<m$ and $\alpha$ the following choices of sequences of movements

$$
\left(u^{i-1} \vee d^{n-i}\right) \bullet\left(l^{j-1} \vee r^{m-j}\right) \vee\left(l^{j-1} \vee r^{m-j}\right) \bullet\left(u^{i-1} \vee d^{n-i}\right)
$$

Proof. Consider the first property, by the adjunctions $-\odot q \dashv[q]-$ and $u^{M} \dashv \square^{M}$, it is equivalent to

$$
u^{M}\left(s_{i j} \odot \alpha\right) \leq s_{11} \vee s_{1 m} \vee s_{n 1} \vee s_{n m}
$$

By join preservation of $\odot$ and $u^{M}$, the above becomes equivalent to showing a join of 8 terms on the left to be less than or equal to the a join of 4 locations on the right. So by definition of join, we must show that all of the following 8 cases hold

$$
\begin{array}{ll}
u^{M}\left(s_{i j} \odot\left(u^{i-1} \bullet l^{j-1}\right)\right) \leq s_{11} & u^{M}\left(s_{i j} \odot\left(u^{i-1} \bullet r^{m-j}\right)\right) \leq s_{1 m} \\
u^{M}\left(s_{i j} \odot\left(d^{n-i} \bullet l^{j-1}\right)\right) \leq s_{n 1} & u^{M}\left(s_{i j} \odot\left(d^{n-i} \bullet r^{m-j}\right)\right) \leq s_{n m} \\
u^{M}\left(s_{i j} \odot\left(l^{j-1} \bullet u^{i-1}\right)\right) \leq s_{11} & u^{M}\left(s_{i j} \odot\left(l^{j-1} \bullet d^{n-i}\right)\right) \leq s_{n 1} \\
u^{M}\left(s_{i j} \odot\left(r^{m-j} \bullet u^{i-1}\right)\right) \leq s_{1 m} & u^{M}\left(s_{i j} \odot\left(r^{m-j} \bullet d^{n-i}\right)\right) \leq s_{n m}
\end{array}
$$

Consider the first one, by uncertainty reduction it suffices to show that

$$
\bigvee\left\{m^{\prime} \mid m^{\prime} \leq u^{M}\left(s_{i j} \cdot\left(u^{i-1} \bullet l^{j-1}\right)\right), m^{\prime} \cdot-1\left(u^{i-1} \bullet l^{j-1}\right) \neq \perp\right\} \leq s_{11}
$$

By associativity of $\odot$ over $\bullet$ and the grid assumptions we have that $s_{i j} \cdot\left(u^{i-1} \bullet l^{j-1}\right)=s_{11}$ and that $u^{M}\left(s_{11}\right)=s_{11}$, also that $s_{11} \cdot{ }^{-1}\left(u^{i-1} \bullet l^{j-1}\right) \neq \perp$, hence the left hand side is equal to $s_{11}$, and trivially we have that $s_{11} \leq s_{11}$. Proofs of the other 7 inequalities are similar.

Now consider the second property $s_{i j} \leq[\alpha] \square^{M}[\alpha]^{-1} s_{i j}$, which is equivalent to the following by adjunction

$$
u^{M}\left(s_{i j} \odot \alpha\right) \odot^{-1} \alpha \leq s_{i j}
$$


Like above $u^{M}\left(s_{i j} \odot \alpha\right)$ breaks down to 8 terms and since $\odot^{-1}$ is also join preserving, one has to show $8 \times 8$ inequalities similar to those in the above, but updated with the $\odot^{-1}$ of the 8 combinations of composition of actions on the left and $s_{i j}$ on the right. That is, we have 8 inequalities of the form

$u^{M}\left(s_{i j} \odot\left(u^{i-1} \bullet l^{j-1}\right)\right) \odot^{-1}\left(\left(u^{i-1} \vee d^{n-i}\right) \bullet\left(l^{j-1} \vee r^{m-j}\right) \vee\left(l^{j-1} \vee r^{m-j}\right) \bullet\left(u^{i-1} \vee d^{n-i}\right)\right) \leq s_{i j}$

We have shown that $u^{M}\left(s_{i j} \odot\left(u^{i-1} \bullet l^{j-1}\right)\right)=s_{11}$, so the above is equivalent to

$$
s_{11} \odot^{-1}\left(\left(u^{i-1} \vee d^{n-i}\right) \bullet\left(l^{j-1} \vee r^{m-j}\right) \vee\left(l^{j-1} \vee r^{m-j}\right) \bullet\left(u^{i-1} \vee d^{n-i}\right)\right) \leq s_{i j}
$$

To show the above, one must show all of the following 8 inequalities

$$
\begin{aligned}
& s_{11} \odot^{-1}\left(u^{i-1} \bullet l^{j-1}\right) \leq s_{i j} \quad s_{11} \odot^{-1}\left(u^{i-1} \bullet r^{m-j}\right) \leq s_{i j} \\
& s_{11} \odot^{-1}\left(d^{n-i} \bullet l^{j-1}\right) \leq s_{i j} \quad s_{11} \odot^{-1}\left(d^{n-i} \bullet r^{m-j}\right) \leq s_{i j} \\
& s_{11} \odot^{-1}\left(l^{j-1} \bullet u^{i-1}\right) \leq s_{i j} \quad s_{11} \odot^{-1}\left(l^{j-1} \bullet d^{n-i}\right) \leq s_{i j} \\
& s_{11} \odot^{-1}\left(r^{m-j} \bullet u^{i-1}\right) \leq s_{i j} \quad s_{11} \odot^{-1}\left(r^{m-j} \bullet d^{n-i}\right) \leq s_{i j}
\end{aligned}
$$

For two of these we have $s_{11} \odot^{-1}\left(d^{n-i} \bullet r^{m-j}\right)=s_{11} \odot^{-1}\left(r^{m-j} \bullet d^{n-i}\right)=s_{i j}$, and the rest are equal to $\perp$ (by the inapplicability assumptions of the grid), which is fine since $\perp$ is less than or equal anything, in particular $s_{i j}$.

\section{Embedding Epistemic Systems}

An algebraic semantics for information learning from communication has been presented in previous work [BCS07], referred to as Epistemic Systems. In this section we make the connection between Epistemic Systems and Nav-diSys formal.

Definition 5.1. A (mono-modal) Epistemic System $(M, Q,-\otimes-, f)$ as defined in [BCS07] is a quantale $Q$ acting on its right module $M$ via the action $-\otimes-: M \times Q \rightarrow M$, where $f=\left(f^{M}: M\right.$ $\left.\rightarrow M, f^{Q}: Q \rightarrow Q\right)$ is a lax system endomorsphism of the setting satisfying the following three inequalities

$$
\begin{aligned}
f^{M}(m \otimes q) & \leq f^{M}(m) \otimes f^{Q}(q) \\
f^{Q}\left(q \bullet q^{\prime}\right) & \leq f^{Q}(q) \bullet f^{Q}\left(q^{\prime}\right) \\
1 & \leq f^{Q}(1)
\end{aligned}
$$

Moreover every element of the quantale $q \in Q$ has a kernel, $\operatorname{ker}(q)=\bigvee\{m \in M \mid m \otimes q=\perp\}$ and the module has a special subset Fact $\subseteq M$, defined as $\Phi=\{p \in M \mid \forall q \in Q, p \otimes q \leq p\}$. The module and quantale have a set of atoms $\operatorname{At}(M)$ and $A t(Q)$ and we have that $A t(M) \subseteq \Phi$.

Inequality number (1) is referred to as the appearance-update inequality. The kernel of each action encodes the propositions to which the action cannot apply, i.e. if you update those propositions with this action, you will get the $\perp$. Kernels are the opposite of the preconditions of actions, as used in the DEL literature, as propositions to which the action can be applied. The facts represent states, and the reason they are stable under updates here is that epistemic actions do not change the state of the world, but only the state of information of agents.

Definition 5.2. An atomic Nav-diSys, similarly atomic Epistemic System, is one that has an atomic module with set of atoms $A t(M)$ and an atomic quantale with a set of atoms $A t(Q)$.

Definition 5.3. A weak reflexive Nav-diSys is an atomic one in which for $s \in A t(M), \pi \in A t(Q)$ we have $s \leq u^{M}(s)$ and $\pi \leq u^{Q}(\pi)^{1}$.

\footnotetext{
${ }^{1}$ Concrete systems that arise from applications have this property.
} 
Theorem 5.4. Given a weak reflexive atomic Nav-diSys $\mathcal{N}$, the structure

$$
\mathcal{N}^{\sigma}=\left(M^{\sigma}, Q^{\sigma},-\otimes-, f\right)_{\Phi}
$$

obtained by setting $M^{\sigma}$ to $M, Q^{\sigma}$ to $Q, f$ to $u$, $\Phi$ to At $(M)$, and $m \otimes q$ to $m \odot q$, is an atomic Epistemic System.

Weak reflexive and transitive Nav-diSys's and Epistemic Systems form a pair of categories with morphisms of each being its corresponding lax endomorphisms. In this setting, the above construction becomes a forgetful functor from the latter to the former, most likely having a right adjoint.

\section{First Steps Towards a Logic}

In order to develop a logic for the algebraic semantics of Nav-diSys, one way would be to start with a logic for converse di-Systems, then add epistemic modalities, and their corresponding interaction axioms to it. The main axiom here would be the uncertainty reduction axiom. The logic of a converse di-System is the positive fragment of Propositional Dynamic Logic with Converse (CPDL) [Par78]. CPDL consists of a propositional and an action logic. To obtain its positive fragment, we must develop positive fragments of each of these logics.

Consider first the propositional part with non-indexed modalities. The syntax of a logic for this part is generated over a set of atomic propositions $p \in P$ via the following grammar:

$$
m::=\perp|\top| p|m \wedge m| m \vee m|\square m| \diamond m\left|\square^{-1} m\right| \diamond^{-1} m
$$

We suggest the following set of axioms and rules as the positive fragment of the propositional part:

Axioms.

$$
\begin{gathered}
m \vdash m, \quad \perp \vdash m, \quad m \vdash \top \\
m \wedge\left(m^{\prime} \vee m^{\prime \prime}\right) \vdash\left(m \wedge m^{\prime}\right) \vee\left(m \wedge m^{\prime \prime}\right) \\
m \vdash m \vee m^{\prime}, \quad m^{\prime} \vdash m \vee m^{\prime}, \quad m \wedge m^{\prime} \vdash m, \quad m \wedge m^{\prime} \vdash m^{\prime} \\
\diamond\left(m \vee m^{\prime}\right) \vdash \diamond m \vee \diamond m^{\prime}, \quad \diamond^{-1}\left(m \vee m^{\prime}\right) \vdash \diamond^{-1} m \vee \diamond^{-1} m^{\prime} \\
\square m \wedge \square m^{\prime} \vdash \square\left(m \wedge m^{\prime}\right), \quad \square^{-1} m \wedge \square^{-1} m^{\prime} \vdash \square^{-1}\left(m \wedge m^{\prime}\right) \\
\top \vdash \square^{-1} \top, \quad \quad \top \vdash \square \top, \quad \diamond \perp \vdash \perp, \quad \diamond^{-1} \perp \vdash \perp
\end{gathered}
$$

Adjunction $\quad \diamond^{-1} \square m \vdash m, \quad m \vdash \square \diamond^{-1} m, \quad \diamond \square^{-1} m \vdash m, \quad m \vdash \square^{-1} \diamond m$

$$
\text { Converse } \quad m \vdash \diamond \diamond^{-1} m, \quad m \vdash \diamond^{-1} \diamond m
$$

Rules.

$$
\begin{aligned}
& \frac{m \vdash m^{\prime} \quad m^{\prime} \vdash m^{\prime \prime}}{m \vdash m^{\prime \prime}} c u t \\
& \frac{m \vdash m^{\prime \prime} \quad m^{\prime} \vdash m^{\prime \prime}}{m \vee m^{\prime} \vdash m^{\prime \prime}} \vee \quad \frac{m \vdash m^{\prime} m \vdash m^{\prime \prime}}{m \vdash m^{\prime} \wedge m^{\prime \prime}} \wedge \\
& \frac{m \vdash m^{\prime}}{\diamond m \vdash \diamond m^{\prime}} \diamond \frac{m \vdash m^{\prime}}{\diamond^{-1} m \vdash \diamond^{-1} m^{\prime}} \diamond^{-1} \quad \frac{m \vdash m^{\prime}}{\square m \vdash \square m^{\prime}} \square \quad \frac{m \vdash m^{\prime}}{\square^{-1} m \vdash \square^{-1} m^{\prime}} \square^{-1}
\end{aligned}
$$


Because of the lack of negation, the axioms and rules are expressed using the notion of a "modal sequent" $m \vdash m^{\prime}$, introduced to deal with positive modal logics, e.g. in [Dun05, GNV05]. The axioms and rules for conjunction and disjunction and normal properties of modalities are obtained from those of a distributive modal lattice from [GNV05]. We have added to this 6 more axioms to capture the adjunction and converse properties of the modalities. In [Par78], only two of our four adjunction axioms are added to the axiomatics of PDL, these are $m \vdash \square \diamond^{-1} m$ and $m \vdash \square^{-1} \diamond m$. In a setting with negation, these suffice, but in our positive setting, it seems that we need all of these to be able to prove completeness.

It is routine to check that the above logical system is sound and complete with regard to the finite nonindexed versions of the axioms of the module of a converse di-System. This follows by taking $m \cdot q$ to be $\diamond m$, and its right adjoint $[q] m$ to be $\square^{-1} m$, also taking $m \cdot{ }^{-1} q$ to be $\diamond^{-1} m$ and its right adjoint $[q]^{-1} m$ to be $\square m$. The apparent swapping of the inverses is because in the logic $\diamond^{-1}$ is the left adjoint to $\square$, whereas in the algebra the right adjoint to $m \cdot q$ is $[q] m$, since adjoints are unique the two have to represent the same formulae ${ }^{2}$.

The syntax of a logic for both the propositional and the action parts is generated over a set of atomic propositions $p \in P$ and a set $\sigma \in \Sigma$ of atomic actions via the following grammar:

$$
\begin{aligned}
m & ::=\perp|\top| p|m \wedge m| m \vee m|[q] m|<q>m\left|[q]^{-1} m\right|<q>^{-1} m \\
q & ::=\top|\perp| \sigma|1| q \bullet q|q \vee q| q^{*}
\end{aligned}
$$

Axioms for the positive fragment of the logic (actions as well as propositions) are the $q$-indexed versions of the previous propositional-only logic, together with the following axioms and rules for action connectives:

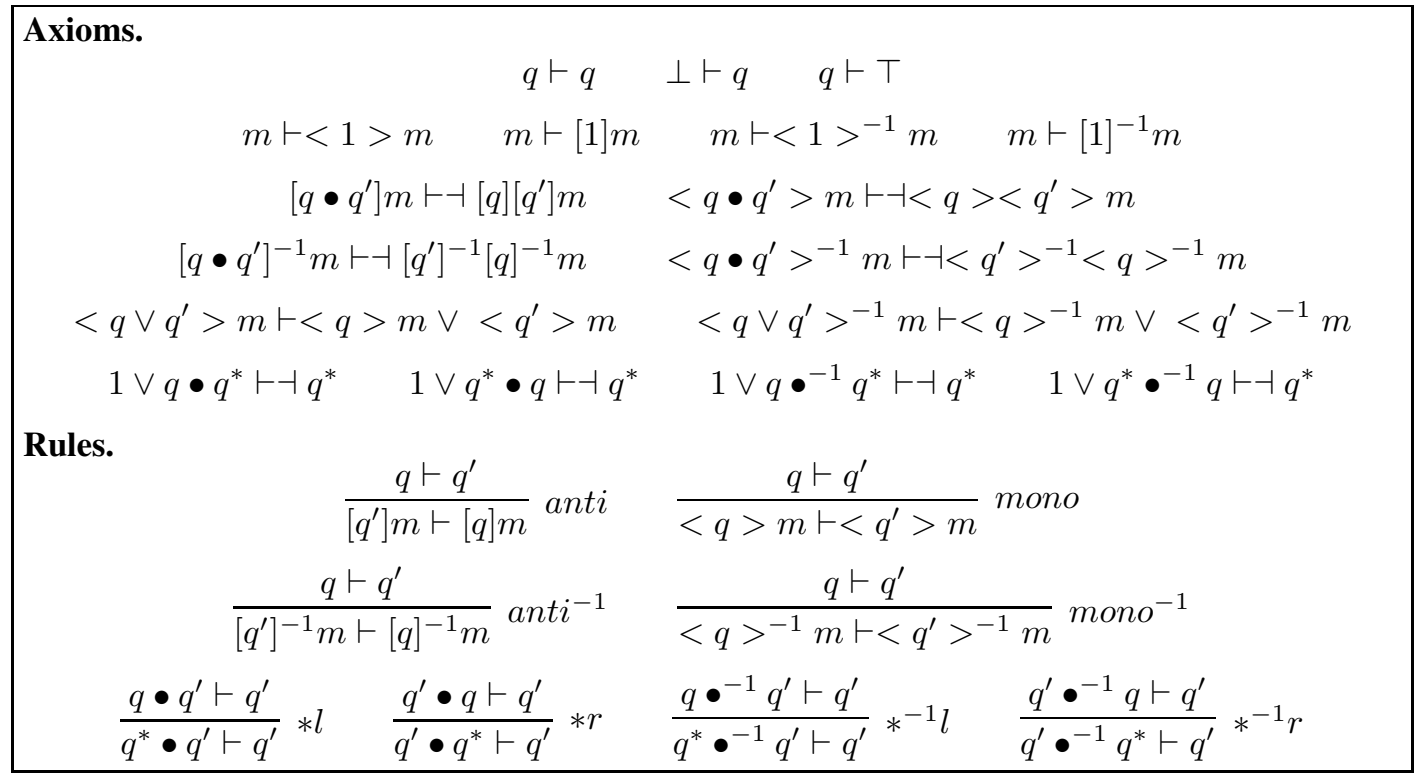

We refer to this logic as $P D L^{+-}$. It is routine to check that it is sound and complete with regard to finite versions of the axioms of a converse di-System, when the actions and their converses are always applicable and once the standard axioms of a Kleene * operator have been added to it. For instance the

\footnotetext{
${ }^{2}$ This is not so surprising, since $[q] m$ stands for the "weakest precondition" of program logics, which has a past tense nature. It represents "all the propositions that must be true before running program $q$, such that proposition $m$ holds after it".
} 
anti rule follows from property (4) of definition 2.4. It is a nice exercise to try and derive the positive versions of axioms of CPDL in our logical system. For example, the proof tree for one direction of axiom (iv) (from p. 173) of [HKT00], i.e. $\left[q \vee q^{\prime}\right] m \vdash[q] m \wedge\left[q^{\prime}\right] m$ is as follows:

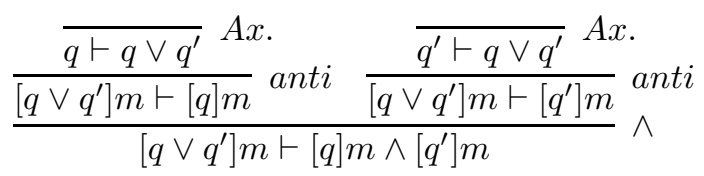

The other direction, i.e. $[q] m \wedge\left[q^{\prime}\right] m \vdash\left[q \vee q^{\prime}\right] m$ is also derivable, but the proof tree is so big that we could not fit it in the page.

The above proof can be generalized to one with regard to converse di-Systems (i.e. with arbitrary joins). This is by showing that the completion of the Lindednbaum-Tarski algebra of $P D L^{+-}$is a converse di-System (with the right assumptions about applicability of actions and axioms for *), and that the embedding of a finite such converse di-system into a converse di-system is a homomorphism. The details mimic the details of the proof of a similar completeness theorem, but for Epistemic Systems, which has been worked out in [Sad06, BCS07].

\section{Conclusions and future work}

We have developed an algebraic framework for dynamic epistemic logic in which the dynamic and epistemic modalities appear as right adjoints. The key new feature in the present work relative to previous work [Sad06, BCS07] is the presence of converse actions and the algebraic laws that govern uncertainty reduction. Robot navigation protocols, as well as the three-player game in Phillips's thesis [Phi09], give examples in which the old learning inequality was violated, showing that there were new subtleties that arise when there are actions that really change the state of the world.

A number of directions for future work naturally suggest themselves. Developing a logic for the full algebra is the main one, establishing closer connections with other DEL's is another. We are also particularly interested in extending this work to apply security protocols where "knowledge" and "learning" play evident roles. A fundamental extension, and one in which we have begun preliminary investigations, is the extension to the probabilistic case. Here knowledge and information theory may well merge in an interesting and not obvious way.

\section{Acknowledgements}

We have benefited greatly from discussions with Caitlin Phillips and Doina Precup. The latter invented the three-player game and the former discovered the violation of the update inequality. This research was supported by EPSRC (UK) and NSERC (Canada) and the Office of Naval Research.

\section{References}

[AV93] S. Abramsky and S. Vickers. Quantales observational logic and process semantics. Mathematical Structures in Computer Science, 3:161-227, 1993. 
[Auch07] G. Aucher and A. Herzig. From DEL to EDL : Exploring the Power of Converse Events. LNCS, 4724: 199-209, 2007.

[BCS07] A. Baltag, B. Coecke, and M. Sadrzadeh. Epistemic actions as resources. Journal of Logic and Computation, 17:555-585, 2007. arXiv:math/0608166.

[DMS06] Jules Desharnais, B. Müller, and G. Struth. Kleene algebra with domain. ACM Trans. Comput. Log., 7:798-833, 2006.

[vanDit05] H.P. van Ditmarsch, W. van der Hoek, B.P. Kooi. Dynamic Epistemic Logic with Assignment. Proceedings of AAMAS, 141-148, 2005.

[Dun05] Michael Dunn. Positive modal logic. Studia Logica, 55:301-317, 2005.

[FHMV95] R. Fagin, J. Y. Halpern, Y. Moses, and M. Y. Vardi. Reasoning About Knowledge. MIT Press, 1995.

[GNV05] M. Gehrke, H. Nagahashi, and Y. Venema. A Sahlqvist theorem for distributive modal logic. Annals of Pure and Applied Logic, 131:65-102, 2005.

[HKT00] D. Harel, D. Kozen, and J. Tiuryn. Propositional Dynamic Logic. MIT Press, 2000.

[HM84] J. Y. Halpern and Y. Moses. Knowledge and common knowledge in a distributed environment. In Proceedings of the Third A.C.M. Symposium on Principles of Distributed Computing, pages 50-61, 1984. A revised version appears as IBM Research Report RJ 4421, Aug., 1987.

[HM90] J. Halpern and Y. Moses. Knowledge and common knowledge in a distributed environment. JACM, 37:549-587, 1990.

[Kri63] S. Kripke. Semantical analysis of modal logic. Zeitschrift fur Mathematische Logik und Grundlagen der Mathematik, 9:67-96, 1963.

[PS10] P. Panangaden and M. Sadrzadeh. Learning in a changing world via algebraic modal logic. http://www.comlab.ox.ac.uk/files/2815/mehrnoosh_prakash.pdf and http://www.cs.mcgill.ca/ prakash/Pubs/mehrnoosh_prakash.pdf

[Par78] R. Parikh. The Completeness of Propositional Dynamic Logic. LNCS 64:403-415, 1978.

[Phi09] C. Phillips. An algebraic approach to dynamic epistemic logic. Master's thesis, School of Computer Sciecne; McGill University, 2009.

[Sad06] M. Sadrzadeh. Actions and Resources in Epistemic Logic. PhD thesis, Université du Québec à Montréal, 2006.

[SD09] M. Sadrzadeh and R. Dyckhoff. Positive logic with adjoint modalities: Proof theory, semantics and reasoning about information. ENTCS 23: 211-225, 2009.

[vDvdHK08] Hans van Ditmarsch, Wiebe van der Hoek, and Barteld Kooi. Dynamic Epistemic Logic. Number 337 in Synthese Library. Springer-Verlag, 2008.

[vK98] B. von Karger. Temporal algebras. Mathematical Structures In Computer Science, 8:277-320, 1998.

[Win09] G. Winskel. Prime algebraicity. Theoretical Computer Science, 410:4160-4168, 2009. 